\title{
Developing Measures of Placement Quality in Allied Health, Dentistry, Medicine, and Pharmacy
}

\author{
Lindy McAllister \\ The University of Sydney, Australia \\ Srivalli Nagarajan \\ The University of Sydney, Australia \\ Linda Scott \\ Formerly of The University of Sydney, Australia \\ Lorraine Smith* \\ The University of Sydney, Australia \\ Kate Thomson \\ The University of Sydney, Australia
}

\begin{abstract}
As placement numbers expand, there is a concern that the quality of student experience and learning may diminish. Furthermore, there is a paucity of evidence for evaluation and quality improvement in clinical health placements and there have been few studies undertaken to assess quality. Valid and reliable measures of placement quality are needed to provide an evidence-base to guide decisions about the most efficient and effective placement models in health. A two-phase mixed methods design, using a modified Delphi process, focus groups, and surveys, developed and tested items to measure placement quality. Thematic analysis, descriptive statistics, and exploratory factor analysis (EFA) were used to analyse the data. Twenty-three participants took part in the Phase 1 stakeholder focus groups, and 150 useable surveys were returned by 161 who took part in Phase 2 to test validity and reliability of the student survey items. Results show broad agreement on the features of a quality clinical placement across allied health dentistry, medicine, and pharmacy professions. The student survey was found to be a valid and reliable measure of placement quality, with the EFA showing one component accounting for $58.5 \%$ of the variance in the survey data. The findings offer a framework and approach that others can adopt to measure placement quality in their setting. The measures may be adaptable to contexts outside health.
\end{abstract}

Keywords: health education; placement quality measures; placements; quality

${ }^{*}$ Corresponding Author: A/Prof Lorraine Smith, The University of Sydney, Faculty of Pharmacy, NSW 2006, Australia

Email: lorraine.smith@sydney.edu.au

Journal URL: http://e-learning.coventry.ac.uk/ojs/index.php/pblh

McAllister, L., Nagarajan, S., Scott, L., Smith, L., and Thomson, K. (2018) 'Developing measures of placement quality in allied health, dentistry, medicine, and pharmacy'. International Journal of Practicebased Learning in Health and Social Care, 6 (2), 31-47

\section{cc) (i) $(\div$}

BY NC ND @ 2018 Lindy McAllister, Srivalli Nagarajan, Linda Scott, Lorraine Smith, Kate Thomson. This Open Access article is distributed under the terms of the Creative Commons Attribution AttributionNon-Commercial No Derivatives 4.0 International License (https://creativecommons.org/licenses/by-nc$\mathrm{nd} / 4.0 /$ ), which permits unrestricted non-commercial use, distribution, and reproduction in any medium, provided the original work is properly cited and is unaltered. 


\section{Introduction}

Work placements are recognised by both graduates and employers as valuable in the development of a range of generic employability skills (Crebert et al. 2004). Clinical or practicebased placements are an essential component of health degrees, and develop disciplinary knowledge and skills, in addition to generic skills. These placements typically comprise onequarter to one-third of the content of allied health degrees (McAllister and Nagarajan 2015). They occur in a range of settings such as hospitals, health centres, schools, private practices, community, disability organisations, and university clinics. Such placements offer students the opportunity to apply theoretical knowledge in a practice context, as well as develop their skills and be socialised into a profession (McCall, Wray, and McKenna 2009). The research reported in this article used the following definition, taken from the Health Workforce Australia (HWA) National Clinical Supervision Support Framework (2011). Placements provide:

... opportunities in a relevant professional setting for the education and training of health sector students for the purposes of (i) Integrating theory into practice; (ii) Familiarising the student with the practice environment; (iii) Building the knowledge, skills and attributes essential for professional practice, as identified by the education institution, and external accrediting/licensing body (HWA 2011: 4)

Globally, there has been considerable growth in numbers of health courses and student enrolments; for example, the number of students enrolled in health-related disciplines leading to professional entry qualifications in Australian universities has doubled in the last decade (HWA 2012). There was a 34\% increase in student enrolments just in physiotherapy courses between 2011 and 2013 (HWA 2014). Given this dramatic expansion and the associated increase in the required volume of placements, there is concern in the health sector regarding the quality of placements (Buchanan, Jenkins, and Scott 2014). Expansion need not threaten quality if there is quality monitoring and management. However, reviews of clinical education and supervision literature show a limited evidence base for evaluation and quality improvement in clinical placements, and few systematic efforts to assess quality in health disciplines (McAllister et al. 2010, Nolte et al. 2011). Important work has been done in establishing valid and reliable measures for evaluating the quality of work-integrated curricula in a range of disciplines (see, for example, Smith 2012). Building on this work, valid and reliable measures of the quality of health student clinical placements are needed to provide an evidence-base to (i) guide decisions about the most efficient and effective models of placement to invest in, (ii) monitor and assure placement quality in health disciplines, and (iii) guide the education, preparation, and support of students and supervisors engaged in clinical placements.

Development of placement quality measures requires an understanding of the features that may contribute to quality. Such features are well described in the Best Practice Clinical Learning Environment (BPCLE) framework (Darcy Associates 2013), which includes six elements: an organisational culture that values learning, best-practice clinical practice, a positive learning environment, an effective health service-education provider relationship, effective communication processes, and appropriate resources and facilities.

The BPCLE framework was developed by Darcy Associates (2013) in response to an Australian state government's request for a framework for excellence in clinical education to enable evaluation of best practice across multiple sites. The online tool associated with the framework is in use in two Australian states; however, there is scant published information on its validity and reliability. There is also insufficient evidence of the robustness of the framework and tool to measure quality across disciplines and stakeholder groups, which is key to identifying placement quality within clinical placements.

Traditionally, measures of clinical placement quality have been either unidisciplinary (e.g. Henzi et al. 2006, Saarikoski et al. 2008, Salamonson et al. 2011, Walters et al. 2011), or focused on a single stakeholder perspective: as examples, students (e.g., Dunn and Burnett 1995, Eley et al. 2015), junior medical staff (e.g., Boor et al. 2007), or supervisors (e.g., Sheils et al. 2016). 
Cusick (2013) found that student learning outcomes were rarely considered in the measurement of placement quality or clinical learning outcomes. Cusick (2013: 9) noted that "an instrument that claims to measure 'quality clinical placements' or 'quality in clinical placements' has not yet been developed or published. Instead, a range of factors have been proposed to be related to quality and a few instruments aim to measure some of these factors". Cusick (2013) suggested that measures based on the BPCLE framework, with the additional element of effective supervision, might be a good approach to developing quality measures (see Figure1). The addition of effective supervision as key to a quality placement is well supported by the literature on clinical education and supervision (Farnan et al. 2012), and so we have adopted Cusick's suggestion in our research.

Figure 1: Proposed features of a quality clinical placement, adapted from the BPCLE framework (Darcy Associates 2013) with the addition of supervision (suggested by Cusick 2013).

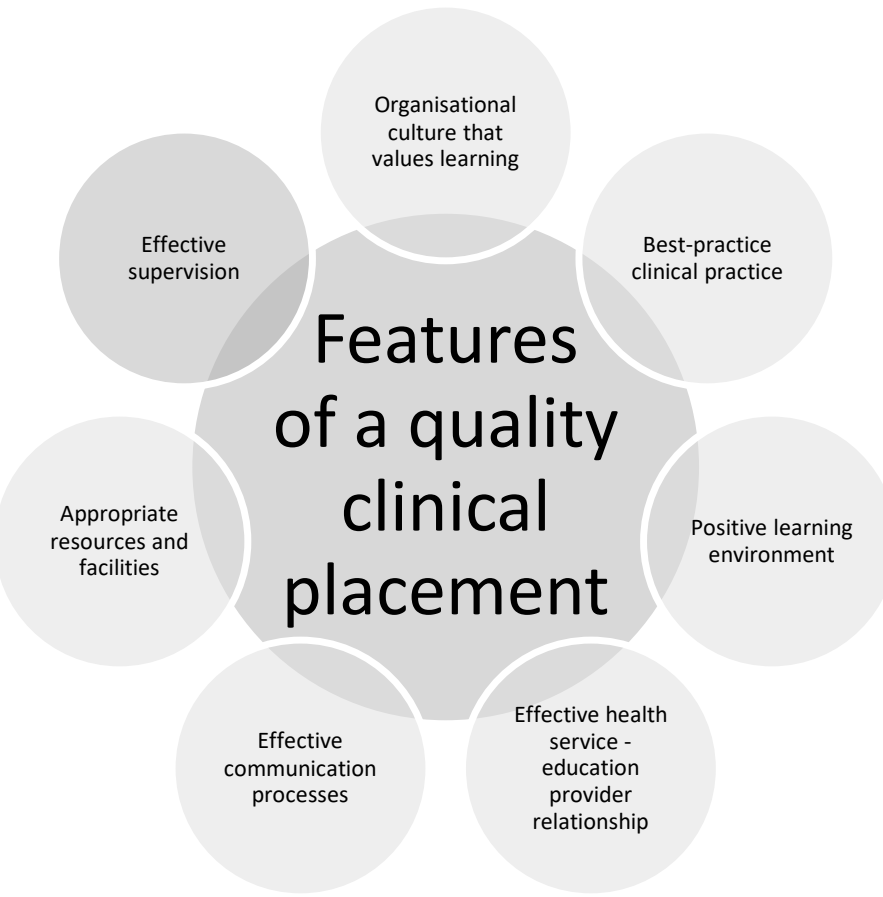

Typically, measures of quality have focused on student evaluation, but students represent one perspective of three key groups in practice-based education, the others being placement sites and education providers (Cooper, Orrell, and Bowden 2010). Additionally, student ratings have been found to be unreliable, with research indicating factors other than quality influence student ratings, such as weather on the day of rating (see Braga, Paccagnella, and Pellizzari 2014, Krautmann and Sander 1999, Poropat 2014). As a result, the research design sought to include multiple stakeholder perspectives to more reliably and holistically measure placement quality.

To capture the features presented in Figure1, a measure needs to include perspectives from each of four stakeholder groups: students, supervisors, placement site managers, and university academics responsible for placements. Perceptions of quality and the meaning of each feature may differ across stakeholder groups. For example, a student may not understand what is meant by 'positive learning environment' in the way that a university academic would. Similarly, a student may report a high quality placement and succeed on placement but their supervisor may report that the student was not independent, and the placement site manager may report that good placement outcomes for the student were achieved at the expense of the supervisor's time for client work, leading to negative impact on departmental client care levels. Evaluating the need for (and usefulness of) a suite of placement quality measures - one for each stakeholder group, which could be compared to ascertain overall placement quality - was deemed important. 
In addition, developing a suite of placement quality measures provides an opportunity for conducting a psychometric assessment of their validity and reliability.

\section{Research aims}

The aims of the research reported in this article were, firstly to test the robustness of the key BPCLE framework features (Darcy Associates 2013), along with Cusick's (2013) suggested additional feature of supervision, and establish whether features needed to be removed or added. The second aim was to develop, pilot, and validate a placement quality measure.

The research questions were:

- How do the features of a quality clinical placement model (Cusick 2013, Darcy Associates 2013) align with stakeholders' perspectives on quality in health placements?

- Could a multi-stakeholder, multidisciplinary approach be used to develop, pilot, and validate measures to evaluate placement quality?

This article reports the outcomes of a two-phase research project which sought to develop and then pilot a suite of placement quality measures that could be used in a range of settings for allied health, dentistry, nursing, and medical students. The article describes the procedures used to develop the measures, pilot them, and assess their reliability and validity. The challenges inherent in this project are described (for example, those associated with a reliance on serial-cascading recruitment of participants), and recommendations provided for others interested in further piloting and refining the measures developed in this research.

\section{Research design}

A sequential exploratory mixed-methods study (Creswell 2003), with two phases: qualitative research followed by quantitative research, was designed to address the aims of the research. Phase 1 used a modified Delphi process and focus groups to achieve consensus on an understanding of quality in health student placements, and to develop surveys for use in Phase 2 , which used online or paper-based surveys and statistical analysis of survey data to test their validity and reliability. The research was approved by the Human Research Ethics committees of the University of Sydney and Sydney Local Health District.

\section{Phase 1: Development of the concept of a quality health student placement}

\section{Method}

\section{Research approach}

We used a modified Delphi technique. The development of the Delphi technique using expert panels to order items in questionnaires is attributed to Dalkey and Helmer (1963). The Delphi technique can include up to four iterations of data collection, in which "responses of previous iterations regarding specific statements and/or items can change or be modified by individual panel members in later iterations based on their ability to review and assess the comments and feedback provided by the other Delphi panelists" (Hsu and Sandford 2007: 2). The aim is to achieve consensus on the inclusion and ranking of items. The Delphi technique has been used extensively in nursing and health sciences to identify and rank priorities (Keeney, Hasson, and McKenna 2011). Delphi panellists traditionally did not engage in discussion of their views with other panellists. We adapted the modified Delphi approach of Dewolfe, Laschinger, and Perkin (2010) who used two rounds of questionnaires followed by a focus group to explore issues for which consensus was not reached. We asked participants to confidentially complete one online survey, participate in a focus group on the features of good and poor quality placements, and then to confidentially complete a second online survey, with the intention of achieving 
consensus by providing participants with the opportunity to revise answers after discussion in the focus group. Consensus was needed to create an agreed-upon survey for validation. Variability between versions of the survey, in addition to existing variability between the categories of respondents would not have allowed for an assessment of validity and reliability, and consequently development of an evidence-based survey. Features for inclusion in the Delphi surveys were taken from the BPCLE framework (Darcy Associates 2013), with Cusick's (2013) suggested addition of supervision.

\section{Participants}

In order to capture comprehensive stakeholder perspectives of quality, via referrals from the study's Project Reference Group, we recruited from four stakeholder groups:

- Students: Students enrolled at the University of Sydney who were currently undertaking, or who had recently undertaken, placements as a compulsory requirement as part of a health degree in the faculties of Education and Social Work, Health Sciences, Medicine, Nursing and Midwifery, and Science;

- University academics: Staff employed by the University of Sydney currently engaged in the organisation of, and support for, student clinical placements in those faculties named above;

- Supervisors: Staff employed in clinical roles, typically by NSW Health ${ }^{1}$, currently or recently involved in the supervision of students enrolled at the University of Sydney in those faculties named above;

- $\quad$ Placement site managers: Staff who manage units, teams or departments within NSW Health that accept students for student placements, but who do not currently directly supervise students themselves. In every case, managers had supervised students in a previous role but their current management responsibilities did not allow them to.

Twenty-three participants from the four stakeholder groups consented to participate in Phase 1: students $(n=5)$, university academics $(n=12)$, supervisors $(n=3)$, and placement site managers $(n=3)$ (see Table 1).

\section{Data collection}

Using a modified Delphi technique, we asked participants to complete an online survey, ranking a number of features of quality clinical placements, and suggesting additional features that they thought important. Two researchers facilitated focus groups: one led the focus group, and one made notes of key points to be used to revise the survey (if needed) for the second Delphi survey. During the focus group, participants discussed their experiences and thoughts on high and low quality clinical placements, and the relevance of each of the features of quality clinical placements that were ranked in the first round of the survey. No new features for addition to the survey were identified in the focus group discussions. Focus groups were digitally recorded for later transcription, analysis, and verification of handwritten notes. At the end of the focus group, participants were again asked to complete the online survey and to rank the features, with the new features identified by individuals in the initial online survey added to the list for the group to consider in their rankings. This process of two opportunities to complete the online survey, with a focus group in between, was repeated for each of the four stakeholder groups. A sample of 11 University staff from the Project Reference Group was used to pilot and refine the methodology

\footnotetext{
${ }^{1}$ NSW Health is the public health system for NSW, a state within Australia, and includes more
} than 230 public hospitals, as well as community and public health services 
and features for inclusion in the Delphi survey, however their contributions were not included in data analysis.

\section{Data analysis}

Data analysis consisted of three stages. Firstly, in addition to features added by individual participants in the initial online survey, notes of focus group discussions were analysed immediately to assess whether items needed to be added to, or deleted from, the second iteration of the Delphi survey. Secondly, to enhance rigour, transcripts of each focus group were reviewed by one of the researchers, and crosschecked with handwritten notes made during the focus groups. No additional features were added on the basis of the focus group discussions, no discrepancies were detected, and the decisions made about the need for revisions to the survey were confirmed. Thirdly, the ranking of items in survey Round 1 were compared with survey in Round 2, with additional features chosen by individuals in Round 1 of the survey considered by all participants in Round 2 (see Tables 2 and $\underline{3}$ ).

Table 1: Survey participants, by discipline and stakeholder group

\begin{tabular}{|l|l|}
\hline Stakeholder group & Discipline \\
\hline Students (5) & Dietetics \\
\cline { 2 - 2 } & Occupational Therapy (3) \\
\cline { 2 - 2 } & Psychology \\
\hline University academics (12) & Health Sciences \\
\cline { 2 - 2 } & Nutrition and Dietetics \\
\cline { 2 - 2 } & Psychology \\
\cline { 2 - 2 } & Work Integrated Learning (9) \\
\hline Supervisors (3) & Physiotherapy \\
\cline { 2 - 2 } & Social Work \\
\cline { 2 - 2 } & Speech Pathology \\
\hline Placement site managers (3) & Occupational Therapy \\
\cline { 2 - 2 } & Physiotherapy (2) \\
\hline Total 23 & \\
\hline
\end{tabular}

\section{Results}

Results from the first round of the modified Delphi process identified broad consensus on the features of a quality placement, and confirmed that all features identified in the BPCLE framework (Darcy Associates 2013), with the addition of supervision, were important.

Delphi findings produced the following averaged rankings (where $1=$ most important feature of quality, and 7 = least important feature of quality), shown in Table 2 below. Specifically, each group ranked the additional feature, effective supervision consistent with the model, as the most important feature of quality clinical placements, with the exception of supervisors themselves who instead ranked it as of less importance than both an organisational culture that values learning, and effective communications processes. Three of the four groups, with the exception of university academics, ranked appropriate resources and facilities as the least or second least important feature of quality. Additional features suggested included: supervisor characteristics; supervision tailored to student needs; ensuring 'match' between student interests, course of 
study, and placement site; and ensuring sufficient clinical exposure to clients for skill development and refinement.

Table 2: Mean first round Delphi rankings of features of a quality clinical placement for all participants (students, university academics, supervisors and site managers)

\begin{tabular}{|l|l|l|l|l|l|}
\hline Features & Overall & Students & $\begin{array}{l}\text { University } \\
\text { academics }\end{array}$ & Supervisors & $\begin{array}{l}\text { Site } \\
\text { Managers }\end{array}$ \\
\hline High quality supervision & 1.67 & 1.6 & 1 & 4 & 1 \\
\hline A positive learning environment & 2.92 & 3 & 2.5 & 5 & 3.7 \\
\hline $\begin{array}{l}\text { An organisational culture that values } \\
\text { learning }\end{array}$ & 3.58 & 3.6 & 6 & 3.5 & 3.3 \\
\hline Effective communication processes & 3.92 & 3 & 5 & 3.5 & 4.7 \\
\hline $\begin{array}{l}\text { An effective health service - } \\
\text { education provider relationship }\end{array}$ & 4.50 & 3.8 & 4.5 & 4.5 & 4 \\
\hline Best practice clinical practice & 5.00 & 5.6 & 3.5 & 4.5 & 5 \\
\hline Appropriate resources and facilities & 5.50 & 5.2 & 2.5 & 4.5 & 6.3 \\
\hline
\end{tabular}

* where 1 = most important feature of quality, and 7 = least important feature of quality

Table 3: Mean second round Delphi rankings of features of a quality clinical placement for all participants.

\begin{tabular}{|l|l|}
\hline Features & $\begin{array}{l}\text { Overall ranking all } \\
\text { participant groups }\end{array}$ \\
\hline Clinical site relevant to one's degree & 1.00 \\
\hline High quality supervision & 2.55 \\
\hline $\begin{array}{l}\text { Supervision tailored to student needs (e.g. balance students taking extra } \\
\text { responsibility with improving areas of poor performance) }\end{array}$ & 2.78 \\
\hline Supervisor characteristics (e.g. approachable) & 3.45 \\
\hline A positive learning environment & 4.55 \\
\hline Relevance to senior student career interests & 4.67 \\
\hline Effective communication processes & 5.64 \\
\hline An effective health service - education provider relationship & 5.91 \\
\hline An organisational culture that values learning & 6.00 \\
\hline Best practice clinical practice & 8.00 \\
\hline Appropriate resources and facilities & 8.27 \\
\hline
\end{tabular}


Following focus group discussion, the second round of the modified Delphi process with additional features produced similar rankings across the four stakeholder groups. The overall feature rankings are shown in Table 3 . Participants continued to place a high degree of importance on the quality of supervision, and the 'match' between the supervision required and the student's needs and interests. In comparison with the first round of results, the only feature to have changed in ranking order was 'An organisational culture that values learning', which moved further towards being considered less important, following the addition of other features focusing on supervision. Again, appropriate resources and facilities were considered the least important feature of quality.

With consensus on the features of quality placements and importance rankings, a draft suite of measures was developed for the four stakeholder groups, for validation in Phase 2 of the study.

\section{Phase 2: Validation of stakeholder measures of a quality student placement}

To establish the robustness of the measures, and provide an opportunity for further refinement of survey items if indicated, descriptive statistics, exploratory factor analysis, and reliability analysis, were undertaken.

\section{Method}

To determine validity and reliability, placement quality measures were designed to be completed twice by participants: once at end of placement, and a second time one week later.

\section{Data collection}

Four placement quality surveys were developed to collect data from each stakeholder group participating in the study. Each survey comprised seven questions; each question was designed to measure features of a student placement, as described in the findings of Phase 1. Questions were answered using a Likert scale, with the anchor points of 1 'extremely poor' levels of quality and 7 'exceptional' levels of quality. The surveys also contained two questions to gather information on perceptions of the quality and utility of the survey. Questions in each of the four surveys used wording to reflect the perspective of the stakeholder completing the survey, and hence there were minor wording variations across the student, university academic, supervisor, and placement site manager surveys. The content focus of each question was the same in all four surveys. In both online and paper versions, surveys took 5 - 10 minutes to complete.

\section{Participants}

Participants were students undertaking compulsory placements as a requirement of their degree in medicine, dentistry, pharmacy, or allied health (physiotherapy, occupational therapy, speech pathology, exercise physiology, diagnostic radiography, nutrition and dietetics, psychology); university academics coordinating placements; supervisors; and placement site managers. We used a cascading recruitment strategy for recruitment of students on placements first, and then for consenting students, we sought to recruit their supervisor, placement site manager and university academic. This recruitment strategy was used to enable linking and triangulation of responses from each of the four stakeholder groups about one learning experience (the same student placement).

To reduce the risk of coercion, students were emailed by a university administration officer and provided with a participant information sheet and consent form to undertake the research. Consenting students were then sent a link to the online survey. Recruitment of students by emails was very slow. They typically did not open the emails or deleted them. After 6 months of recruitment activity, only seven students had consented, so modifications were made, following approval from the relevant ethics body. Incentives for students were introduced, and a placement site administrator approached students to provide them with a participant information 
sheet and consent form, and paper versions of the surveys were introduced as an alternative to the online version already available

Five hundred and ninety-eight emails were sent to eligible students at ethics-approved sites within the 12-month study period. The number of recruitment emails sent and the number of students recruited for each discipline is shown in Table 4 below. One hundred and sixty-one students were recruited and completed Survey 1, a response rate of $27 \%$ (ranging from $16 \%$ to $51 \%$ across the disciplines). The majority of students were from Allied health ${ }^{2}(n=56)$, and Dentistry $(n=52)$, followed by Pharmacy $(n=34)$, Medicine $(n=18)$, and Unknown $(n=1)$.

There was no response to recruitment emails sent to supervisors or managers of students who had consented, that is, those staff in hospitals who supervised student placements and managed placement sites. Consequently, refinement of the measures was not possible for these groups. Eight surveys were returned from university academics coordinating placements. To examine test-retest reliability, all participants who completed Survey 1 (8 academics and 161 students) were invited to complete the same survey one week later. Test-retest reliability is important because the intention is that the surveys will be used as repeated measures of placement quality, and to collect data that are consistent across different points in time. The time period between first and second administrations varies widely, but to reduce the possibility of confounding factors (such as students attending another placement immediately after the placement they completed the survey for) and increase the likelihood of only random error occurring, a one-week time period between the two survey administrations was selected (Salkind 2010). Only 27 students and no university academics completed the survey a second time, and so test-retest reliability is available for the student survey.

\section{Results}

\section{Student ratings of placement quality}

The focus of this section is on the survey data collected from students, one of the four stakeholder groups. Data analysis was conducted on the 150 useable surveys returned by 161 students. Mean and median Likert scale figures were calculated for each question. Results show that participants utilised the range of possible responses, however responses clustered to the positive end of the scale (see Table 4). No responses of the lowest rating of quality (1) were utilised by any participant, and all seven questions had a mean ratings of greater than five, ranging from 5.13 to 5.79. All questions had a median response of six, with the exception of 'Levels of communication between the university and the site', which had a median response of five.

For the additional two questions seeking information on the quality and usefulness of the surveys, means and medians showed the surveys were consistently rated as being of high quality and useful, with both questions receiving mean and median ratings of 5 , where 1 was 'extremely poor' levels of quality/usefulness and 7 'exceptional' levels of quality/usefulness.

\footnotetext{
2 Diagnostic radiography, nutrition and dietetics, occupational therapy, physiotherapy, psychology, speech pathology
} 
Table 4: Responses by Questions - Clinical Placement Quality*

\begin{tabular}{|l|l|l|}
\hline Question & Mean Response & Median Response \\
\hline $\begin{array}{l}\text { Overall, how would you rate the quality of this } \\
\text { placement? }\end{array}$ & 5.69 & 6.00 \\
\hline $\begin{array}{l}\text { Overall, how would you rate the fit between the } \\
\text { placement site and supervision you received } \\
\text { and your learning needs? }\end{array}$ & 5.72 & 6.00 \\
\hline $\begin{array}{l}\text { Overall, how would you rate the quality of the } \\
\text { supervision you received? }\end{array}$ & 5.79 & 6.00 \\
\hline $\begin{array}{l}\text { Overall, how would you rate the quality of the } \\
\text { learning environment in the workplace? }\end{array}$ & 5.70 & 6.00 \\
\hline $\begin{array}{l}\text { Overall, how would you rate the level of } \\
\text { communication between your university and } \\
\text { the site of your clinical placement? }\end{array}$ & 5.13 & 5.00 \\
\hline $\begin{array}{l}\text { Overall, how would you rate the organisational } \\
\text { culture of learning at the site of your clinical } \\
\text { placement? }\end{array}$ & 5.48 & 6.00 \\
\hline $\begin{array}{l}\text { Based on your knowledge, was the suite of } \\
\text { practice skills and clinical reasoning you were } \\
\text { taught best practice clinical practice and } \\
\text { knowledge? }\end{array}$ & 5.76 & 6.00 \\
\hline
\end{tabular}

*Where 1 was 'extremely poor' quality and 7 'exceptional' quality

\section{Survey factor structure}

Exploratory factor analysis was undertaken to examine the extent to which the seven items in the survey, previously determined through the modified Delphi process and focus groups, would adequately explain the underlying concept of clinical placement quality.

Figure 2: Scree plot for Exploratory Factor Analysis - clinical placement quality

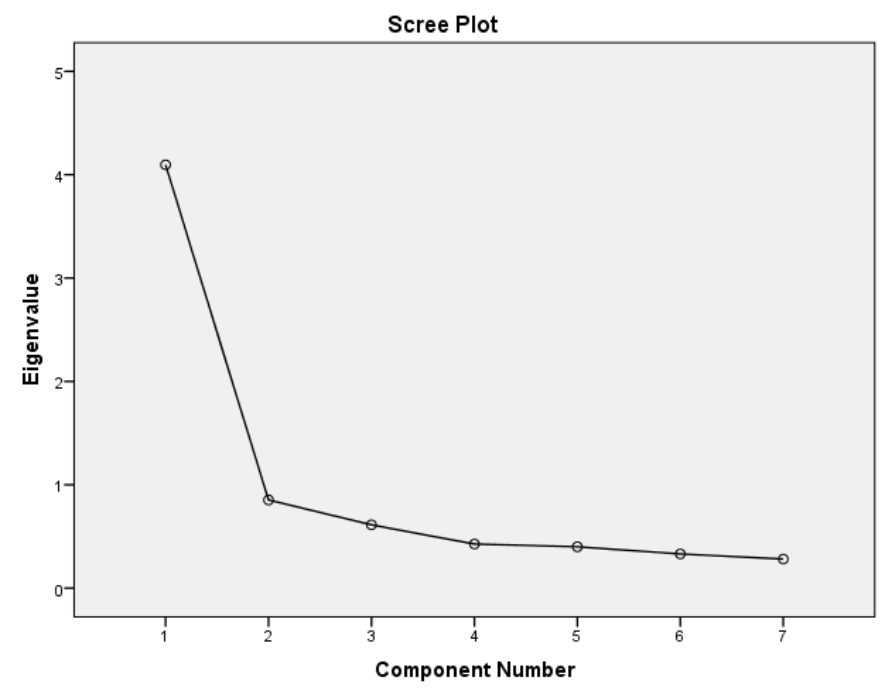


Using a sample size of $N=150$, the Bartlett Test of Sphericity was significant and the KaiserMeyer-Olkin (KMO) measure of sampling adequacy was 0.88. One component (with eigenvalue $>1$ ) was extracted accounting for $58.5 \%$ of the variance. The eigenvalue was 4.10 . The Scree Plot (Figure 2) indicates one component factor emerging. The resulting component matrix is displayed below in Table 5 .

Table 5: Component matrix for Exploratory Factor Analysis - clinical placement quality

\begin{tabular}{|l|l|}
\hline Variable & Component \\
\hline & $\mathbf{1}$ \\
\hline Overall, how would you rate the quality of this placement? & 0.79 \\
\hline $\begin{array}{l}\text { Overall, how would you rate the fit between the placement site and } \\
\text { supervision you received and your learning needs? }\end{array}$ & 0.81 \\
\hline $\begin{array}{l}\text { Overall, how would you rate the quality of supervision you received? } \\
\text { Overall, how would you rate the quality of the learning environment in the } \\
\text { workplace? }\end{array}$ & 0.79 \\
\hline $\begin{array}{l}\text { Overall, how would you rate the level of communication between your } \\
\text { university and the site of your clinical placement? }\end{array}$ & 0.67 \\
\hline $\begin{array}{l}\text { Overall, how would you rate the organisational culture of learning at the } \\
\text { site of your clinical placement? }\end{array}$ & 0.74 \\
\hline $\begin{array}{l}\text { Based on your knowledge, was the suite of practice skills and clinical } \\
\text { reasoning you were taught best practice clinical practice and knowledge? }\end{array}$ & 0.72 \\
\hline
\end{tabular}

The internal consistency of the items, as measured by Cronbach's alpha, was 0.87 . Removal of any items would result in poorer internal consistency. Taken together, these results indicate a robust factor structure of the survey.

\section{Test-retest reliability}

Bivariate correlations were conducted to measure the reliability of the surveys over time. Included in the analysis were the 7 questions relating to quality of clinical placements, and 2 questions relating to survey quality and usefulness. Reliability coefficients ranged from 0.52 to 0.85 , indicating a range of poor to very good reliability, as shown in Table 6.

\section{Discussion}

Drawing on a model of the features of a high-quality clinical placement, we have used a multistakeholder approach to develop and test measures of placement quality in health disciplines. In a context where placement volume and settings are expanding, these measures are intended to contribute an evidence base for: designing and advancing placement models, assuring and enhancing the quality of placement experiences, and informing decisions around resources for training and assistance for students and supervisors (HWA 2012, 2014, Buchanan, Jenkins, \& Scott 2014). 
Table 6: Test-retest reliability coefficients

\begin{tabular}{|l|l|l|}
\hline Variable & Coefficient & Significance level \\
\hline $\begin{array}{l}\text { Overall, how would you rate the quality of this } \\
\text { placement? }\end{array}$ & 0.85 & $<0.001$ \\
\hline $\begin{array}{l}\text { Overall, how would you rate the fit between the } \\
\text { placement site and supervision you received and } \\
\text { your learning needs? }\end{array}$ & 0.85 & $<0.001$ \\
\hline $\begin{array}{l}\text { Overall, how would you rate the quality of } \\
\text { supervision you received? }\end{array}$ & 0.58 & $<0.001$ \\
\hline $\begin{array}{l}\text { Overall, how would you rate the quality of the } \\
\text { learning environment in the workplace? }\end{array}$ & 0.74 & $<0.001$ \\
\hline $\begin{array}{l}\text { Overall, how would you rate the level of } \\
\text { communication between your university and the } \\
\text { site of your clinical placement? }\end{array}$ & 0.69 & $<0.001$ \\
\hline $\begin{array}{l}\text { Overall, how would you rate the organisational } \\
\text { culture of learning at the site of your clinical } \\
\text { placement? }\end{array}$ & 0.55 & 0.003 \\
\hline $\begin{array}{l}\text { Based on your knowledge, was the suite of } \\
\text { practice skills and clinical reasoning you were } \\
\text { taught best practice clinical practice and } \\
\text { knowledge? }\end{array}$ & 0.54 & 0.004 \\
\hline $\begin{array}{l}\text { Please rate the quality of the survey you are } \\
\text { currently completing }\end{array}$ & 0.52 & $<0.001$ \\
\hline $\begin{array}{l}\text { Please rate the usefulness of the survey you are } \\
\text { currently completing }\end{array}$ & 0.71 & \\
\hline
\end{tabular}

In Phase 1, we found there was broad consensus on the features of quality clinical placements, and all BPCLE framework features, with the addition of quality supervision, were important. While low sample sizes do not allow for statistical analysis of rankings, there is broad agreement between stakeholder groups that the quality of supervision is a critical determinant of the quality of a clinical placement and that the material resources for students while on placement, while important, are less important than other features. Specifically, each group ranked supervision as the most important feature of quality clinical placements, with the exception of supervisors themselves who instead ranked it after an organisational culture that values learning, and effective communication. This recognition of the key role of supervisors means that at sites where these measures (or other data sources) identify a need to improve the quality of clinical placements, strategies should seek to develop supervisors and foster an organisational culture that values learning (Higgs and McAllister 2007, Thomson, Nguyen, and Leithhead 2016). Additional features of quality clinical placements included supervisor characteristics, supervision tailored to student needs, ensuring 'match' between student interests, course of study and placement site, and ensuring sufficient clinical exposure to clients for skill development and refinement. This leads to additional questions around what combinations of features might create the highest quality clinical placement and what is appropriate for quality enhancement; for example, whether and how resources should be invested in tailoring supervision or matching student interests and placement experiences. The 
research also confirmed the appetite for a placement quality measure - a suite of placement quality surveys, and for data to support future refinement of clinical placements to improve quality.

As a result of this analysis, a suite of surveys was developed for piloting, validation, and reliability testing in Phase 2, using a range of sites to capture diversity in placement types and quality.

In Phase 2, we found that the seven items used in the surveys adequately captured placement quality, and were a robust reflection of the clinical placement survey construct, as indicated by the exploratory factor analysis results. A limitation of this phase is that the surveys were not completed by the students' supervisors and placement site managers, and only by eight university academics, so a multi-stakeholder measure of placement quality remains to be confirmed. Further research is required to evaluate the factor structure, validity, and reliability of the measures for the other stakeholder groups; supervisors, placement site managers, and university academics. Our approach to recruitment relied on students primarily because without their consent the cascading recruitment of other stakeholders could not occur. Should wider implementation occur, this approach needs additional administrative support to recruit other stakeholders which can be resource intensive and time consuming. One suggestion to overcome this challenge is to incorporate quality surveys as a routine component of students' activities during their last week of placement. Placing emphasis on the importance of student participation in completing these surveys during placement briefing and preparation sessions provided by the disciplines should be considered.

The difficulties encountered in engaging supervisors and placement site managers in completing the quality surveys highlights the challenges involved in implementing these surveys at placement sites. Increased participation of supervisors and managers is only possible when placement sites work collaboratively with university academics to implement the measures and disseminate the benefits for them in systematically implementing and using these surveys (for example, data arising from these surveys could inform sites' quality improvement processes). Currently, supervisors and placement site managers do not evaluate the quality of placement using valid and reliable measures (McAllister et al. 2010, Nolte et al. 2011), and their perspectives on placement quality may at best be shared informally with university academics through phone or email correspondence. Supervisors' roles in placements mean that their perspective provides key evidence for both assuring placement quality and identifying appropriate preparation and support to improve placement experiences for students and supervisors. The perspective of site managers informs our understanding of which placement models are efficient and effective, and consequently, which should be promoted. The impact of evidence-based decisions around quality assurance, supervisor support programs, and placement models, on overall student learning and the quality of degree programs increases with placement growth (HWA 2012, 2014, Buchanan, Jenkins, and Scott 2014). Without data gathered through valid and reliable measures, the evidence used for these decisions may not be appropriate and will vary across sites and over time. One recommendation as part of implementation of these measures is to disseminate and formally train site supervisors and managers through existing or new supervisor development activities. These are significant procedural changes which call for a systemic level collaboration and cooperation from the disciplines, faculties, universities as a whole, and placement sites and supervisors.

\section{Conclusion}

An evaluation of placement quality needs to clearly indicate areas for improvement across each feature (for example, a positive learning environment, effective communication processes, and appropriate resources and facilities: see Figure 1 for all features). Such an evaluation would allow for longitudinal benchmarking to compare and evaluate outcomes against quality improvement strategies. Drawing on an adapted version of a best practice framework, our study provides a way for measuring placement quality using a multi-stakeholder approach. We have 
developed and tested what appears to be the first multidisciplinary measure of placement quality, in an attempt to overcome the narrow foci of previous placement quality measures (see for example Henzi et al. 2006, Saarikoski et al. 2008, Salamonson et al. 2011, Walters et al. 2011). The student survey appears to overcome issues of reliability previously noted (Braga, Paccagnella, and Pellizzari 2014, Poropat 2014). The study findings add to a growing body of literature on understanding how to measure placement quality using a robust suite of measures. We have devised a methodology which can be replicated or adapted by other providers seeking to measure and improve quality of placements. Further studies, which include larger samples of stakeholder groups, will need to be undertaken to establish validity and reliability of the developed quality measures. We suggest that these surveys and the methodology are applicable to development and testing of placement quality measures in disciplines outside health. Whatever measure of quality is adopted, concerns will remain about definitions, benchmarking, and the relationship between assessment and improvement. These measures are designed to clearly indicate features for improvement, and allow for a comparison of outcomes against local and sector-wide reforms.

\section{Acknowledgements}

This work was supported by the NSW Health Education and Training Institute (HETI), the Sydney Interdisciplinary Clinical Training Network (ICTN), and the University of Sydney Large Education Innovation grant scheme 2014.

We gratefully acknowledge members of the Project Reference Group. 


\section{References}

Boor, K., Scheele, F., van der Vleuten, C.P., Scherpbier, A.J., Teunissen, P.W., and Sijtsma, K. (2007) 'Psychometric properties of an instrument to measure the clinical learning environment'. Medical Education, 41, 92-99 http://dx.doi.org/10.1111/i.13652929.2006.02651.x

Braga, M., Paccagnella, M., and Pellizzari, M. (2014) 'Evaluating students' evaluations of professors'. The Economics of Education Review, 41, 71-88 https://doi.org/10.1016/i.econedurev.2014.04.002

Buchanan, J., Jenkins, S., and Scott, L. (2014) Student Clinical Education in Australia: A University of Sydney Scoping Study. Sydney: The University of Sydney

Cooper, L., Orrell, J., and Bowden, M. (2010) Work Integrated Learning: A Guide to Effective Practice. Abingdon, UK: Routledge

Crebert, G., Bates, M., Bell, B., Patrick, C.-J., and Cragnolini, V. (2004) 'Developing generic skills at university, during work placement and in employment: Graduates' perceptions'. Higher Education Research \& Development, 23, 147-165. http://dx.doi.org/10.1080/0729436042000206636

Creswell, J.W. (2003) Research Design: Qualitative, Quantitative, and Mixed Method Approaches. 2nd edn. London: Sage

Cusick, A. (2013) Measuring Quality Clinical Placements: Literature Review. Final Report. Sydney: Integrated Clinical Training Network (ICTN)

Dalkey, N. and Helmer, O. (1963) 'An experimental application of the Delphi method to the use of experts'. Management Science, 9, 458-467 https://doi.org/10.1287/mnsc.9.3.458

Darcy Associates (2013) The Best Practice Clinical Learning Environment Framework. Quality Clinical Education in Victoria. Available from https://www.bpcletool.net.au/bpcleframework/ [6 June 2018]

Dewolfe, J.A., Laschinger, S., and Perkin, C. (2010) 'Preceptors' perspectives on recruitment, support, and retention of preceptors'. Journal of Nursing Education, 4, 198-206 https://doi.org/10.3928/01484834-20091217-06

Dunn, S.V. and Burnett, P. (1995) 'The development of a clinical learning environment scale'. Journal of Advanced Nursing, 22, 1166-1173 https://doi.org/10.1111/j.13652648.1995.tb03119.x

Eley, D.S., McAllister, L., Chipchase, L., Strong, J., Allen, S., and Davidson, B. (2015) 'Health professions as distinct cultures in interprofessional, intercultural clinical placements: A pilot study exploring implications for interprofessional supervision'. International Journal of Practice-based Learning in Health and Social Care, 3 (1), 108-118 http://dx.doi.org/10.18552/iipblhsc.v3i1.211

Farnan, J., Petty, L., Georgitis, E., Martin, S., Chiu, E., Prochaska, M., and Arora, V. (2012) 'A systematic review: The effect of clinical supervision on patient and residency education outcomes'. Academic Medicine, 87, 4, 428-442 https://doi.org/10.1097/ACM.0b013e31824822cc

Health Workforce Australia. (2011) National Clinical Supervision Support Framework. Adelaide: Health Workforce Australia 
Health Workforce Australia. (2012) Health Workforce 2025 - Doctors, Nurses and Midwives. Volume 1. Adelaide: Health Workforce Australia. Available from http://www.health.gov.au/internet/main/publishing.nsf/Content/hwa-archivedpublications [6 June 2018]

Health Workforce Australia. (2014) Australia's health workforce series: Physiotherapists in focus. Adelaide: Health Workforce Australia.

Henzi, D., Davis, R., Jasinevicius, R., and Hendricson, W. (2006) 'North American dental students' perspectives about their clinical education'. Journal of Dental Education, 70, 361-377

Higgs, J. and McAllister, L. (2007) 'Educating clinical educators: Using a model of the experience of being a clinical educator'. Medical Teacher, 29 (2-3), e51-e57 https://doi.org/10.1080/01421590601046088

Hsu, C.-C. and Sandford, B.A. (2007) 'The Delphi technique: Making sense of consensus'. Practical Assessment, Research and Evaluation, 12 (10), 1-8

Keeney, S., Hasson, F., and McKenna, H. (2011) The Delphi Technique in Nursing and Health Research. Oxford: Wiley-Blackwell https://doi.org/10.1002/9781444392029

Krautmann, A. and Sander, W. (1999) 'Grades and student evaluations of teachers'. Economics of Education Review, 18, 59-63 https://doi.org/10.1016/S0272-7757(98)00004-1

McAllister, L. and Nagarajan, S.V. (2015) 'Accreditation requirements in allied health education: Strengths, weaknesses and missed opportunities. Journal of Teaching and Learning for Graduate Employability, 6, 2-23 http://dx.doi.org/10.21153/jtlge2015vol6no1art570

McAllister, L., Paterson, M., Higgs, J., and Bithell, C. (2010) Innovations in allied health fieldwork education: A critical appraisal. Rotterdam: Sense

McCall, L., Wray, N., and McKenna, L. (2009) 'Influence of clinical placement on undergraduate midwifery students' career intentions'. Midwifery, 25, 403-410 https://doi.org/10.1016/j.midw.2007.07.008

Nolte, E., Fry, C.V., Winpenny, E., and Brereton, L. (2011) Use of Outcome Metrics to Measure Quality in Education and Training of Healthcare Professionals: A Scoping Review of International Experiences. Department of Health in England through its Policy Research Programme (grant no. 0510002). Cambridge, UK: RAND Europe working paper series

Poropat, A. (2014) 'Students don't know what's best for their own learning'. The Conversation [online] 26 November. available from http://theconversation.com/students-dont-knowwhats-best-for-their-own-learning-33835[30 May 2018]

Saarikoski, M., Isoaho, H., Warne, T., and Leino-Kilpi, H. (2008) 'The nurse teacher in clinical practice: Developing the new sub-dimension to the clinical learning environment and supervision (CLES) scale'. International Journal of Nursing Studies, 45, 1233-1237 https://doi.org/10.1016/.ijnurstu.2007.07.009

Salamonson, Y., Bourgeois, S., Everett, B., Weaver, R., and Peters, K. (2011) 'Psychometric testing of the abbreviated Clinical Learning Environment Inventory (CLEI-19)'. Journal of Advanced Nursing, 67, 2668-2676 https://doi.org/10.1111/j.1365-2648.2011.05704.x 
Salkind, N. (2010) Encyclopedia of Research Design. Thousand Oaks, CA: Sage https://doi.org/10.4135/9781412961288

Sheils, E. A., Loades, M. E., Medley, A. R., and Marks, E.M. (2016) 'A fair exchange: The reciprocal relationship between universities and clinical placement supervisors'. International Journal of Practice-based Learning in Health and Social Care, 4 (1), 28-39 https://doi.org/10.18552/ijpblhsc.v4i1.304

Smith, C. (2012) 'Evaluating the quality of work-integrated learning curricula: A comprehensive framework'. Higher Education Research \& Development, 31, 247-262 http://dx.doi.org/10.1080/07294360.2011.558072

Thomson, K., Nguyen, M., and Leithhead, I. (2016) 'Peer mentoring for clinical educators: A case study in physiotherapy'. Focus on Health Professional Education, 17 (3), 30-44 https://doi.org/10.11157/fohpe.v17i3.175

Walters, L., Prideaux, D., Worley, P., and Greenhill, J. (2011) 'Demonstrating the value of longitudinal integrated placements to general practice preceptors'. Medical Education, 45, 455-463 https://doi.org/10.1111/j.1365-2923.2010.03901.x 